flow, including wall shear stress and oscillatory shear index, correlated with EB gene expression and supported evidence of vascular remodeling.

Conclusions EB is a safe technique to permit non-invasive genome-wide sequencing of bAVMs. Transcriptomic data correlates well with tissues acquired with open surgery and implicates Ras-MAPK signaling in adult bAVMs. It is also now possible to correlate gene expression with CFD flow modeling in living patients. Further development of noninvasive diagnostic techniques to molecularly stratify patients are needed to better delineate divergent natural histories of bAVMs and potentially inform treatment planning.

Disclosures E. Winkler: None. D. Wu: None. D. McCoy: None. A. Abla: None. D. Cooke: None.
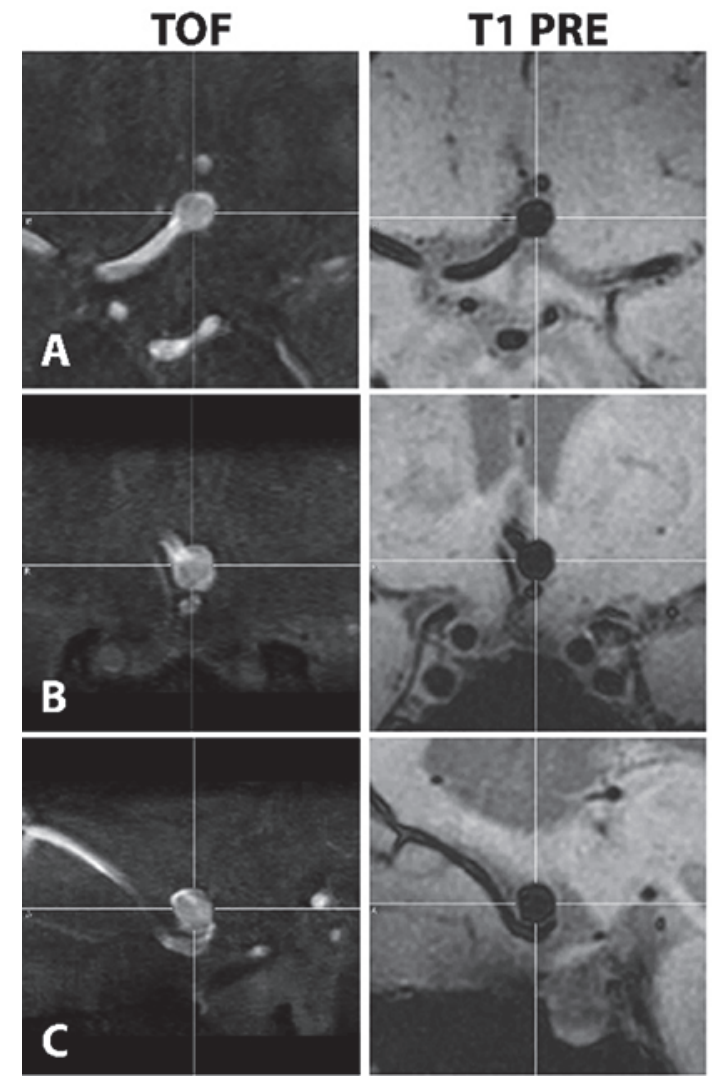

\section{0-021 INCREASED CONTRAST ENHANCEMENT OF THE PARENT VESSEL OF UNRUPTURED INTRACRANIAL ANEURYSMS IN 7T MR IMAGING}

${ }^{1}$ E Samaniego*, ${ }^{2} \mathrm{~J}$ Roa, ${ }^{3} \mathrm{H}$ Zhang, ${ }^{4} \mathrm{~T}$ Koscik, ${ }^{1} \mathrm{~S}$ Ortega-Gutierrez, ${ }^{5} \mathrm{G}$ Bathla, ${ }^{3} \mathrm{M}$ Sonka, ${ }^{5} \mathrm{C}$ Derdeyn, ${ }^{5} \mathrm{~V}$ Magnotta, ${ }^{6} \mathrm{D}$ Hasan. ${ }^{1}$ Neurology, Neurosurgery and Radiology, University of lowa Hospitals and Clinics, lowa City, IA; ${ }^{2}$ Neurology and Neurosurgery, University of lowa Hospitals and Clinics, lowa City, IA; ${ }^{3}$ Electrical and Computer Engineering, lowa Institute of Biomedical Imaging, University of lowa Hospitals and Clinics, lowa City, IA; ${ }^{4}$ sychiatry, University of lowa Hospitals and Clinics, lowa City, IA; ${ }^{5}$ Radiology, University of lowa Hospitals and Clinics, lowa City, IA; ${ }^{6}$ Neurosurgery, University of lowa Hospitals and Clinics, lowa City, IA

\subsection{6/neurintsurg-2020-SNIS.21}

Background Inflammation of the cerebral arterial wall may lead to aneurysm formation. The presence of aneurysm

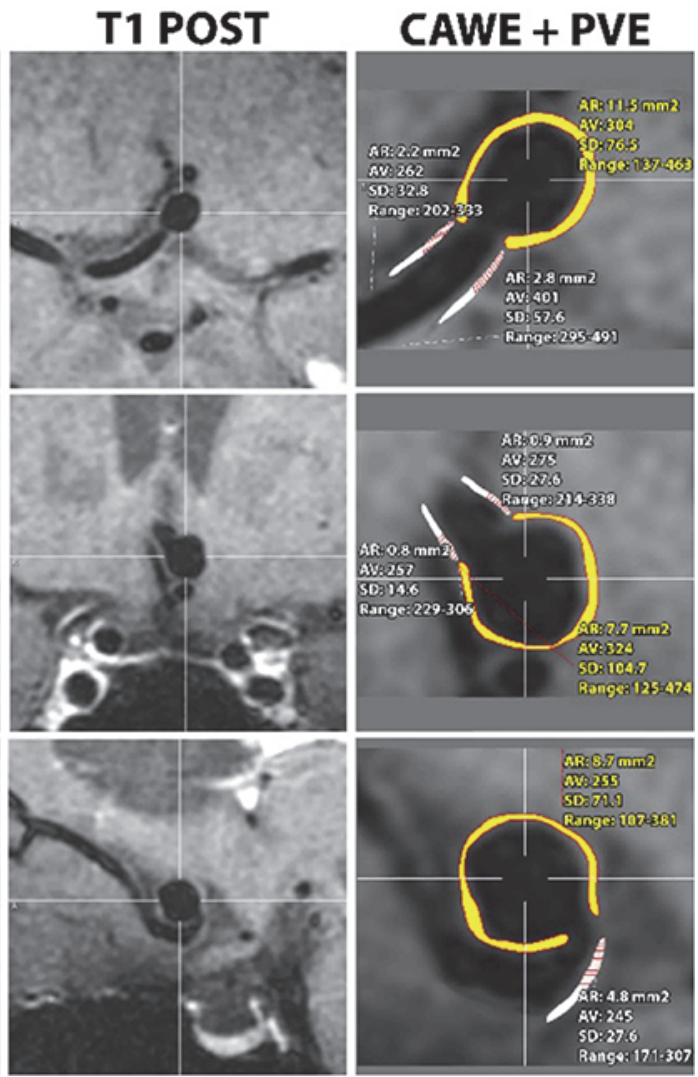



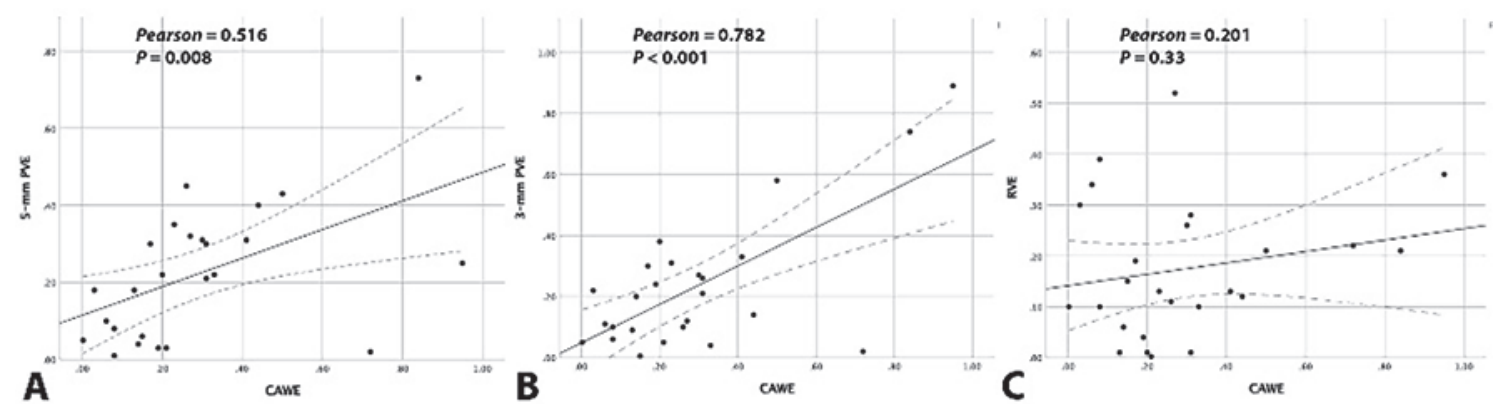

\section{Abstract 0-021 Figure 2}

enhancement on high-resolution vessel wall imaging (HR-VWI) is a marker of wall inflammation and instability. We aim to determine if there is any association between increased contrast enhancement in the aneurysmal wall and its parent artery using 7T HR-VWI.

Methods Patients with unruptured intracranial aneurysms (UIAs) underwent 7T HR-VWI. Regions of interest were selected manually and with a semi-automated protocol based on gradient algorithms of intensity patterns. Mean signal intensities in pre- and post-contrast T1-weighted sequences were adjusted to the enhancement of the pituitary stalk and then subtracted to objectively determine: 1) circumferential aneurysmal wall enhancement (CAWE), 2) parent vessel enhancement (PVE), and 3) reference vessel enhancement (RVE). PVE was assessed over regions located 3- and 5-mm from the aneurysm's neck. RVE was assessed in arteries located in a different vascular territory.

Results Twenty-five UIAs were analyzed. There was a significant moderate correlation between CAWE and 5-mm PVE (Pearson $\mathrm{R}=0.52, \quad P=0.008$ ), whereas no correlation was found between CAWE and RVE (Pearson $\mathrm{R}=0.20, P=0.33$ ). A stronger correlation was found between CAWE and 3-mm PVE (Pearson $\mathrm{R}=0.78, P<0.001$ ). Intra-class correlation analysis demonstrated good reliability between measurements obtained using semi-automated and manual segmentation (ICC coefficient $=0.790,95 \%$ CI $0.58-0.90)$.

Conclusion Parent arteries exhibit higher contrast enhancement in regions closer to the aneurysm's neck, especially in aneurysms $\geq 7 \mathrm{~mm}$. A localized inflammatory/vasculopathic process in the wall of the parent artery may lead to aneurysm formation and growth.
Disclosures E. Samaniego: 1; C; SVIN 2019, Bee Foundation 2019. 2; C; Medtronic, MicroVention. J. Roa: None. H. Zhang: None. T. Koscik: None. S. Ortega-Gutierrez: 2; C; Stryker, MicroVention. G. Bathla: None. M. Sonka: None. C. Derdeyn: None. V. Magnotta: None. D. Hasan: None.

\section{0-022 DUAL ANTIPLATELET THERAPY AFTER CAROTID ARTERY STENTING: TRENDS AND OUTCOMES IN A LARGE NATIONAL DATABASE}

${ }^{1}$ E Sussman*, ${ }^{2} \mathrm{M}$ Jin, ${ }^{1} \mathrm{~A}$ Pendharkar, ${ }^{3} \mathrm{~B}$ Pulli, ${ }^{2} \mathrm{~A}$ Feng, ${ }^{3} \mathrm{~J}$ Heit, ${ }^{3} \mathrm{~N}$ Telischak. ${ }^{1}$ Neurosurgery, Stanford University, Stanford, CA; ${ }^{2}$ Stanford University, Stanford, CA; ${ }^{3}$ Radiology, Stanford University, Stanford, CA

\subsection{6/neurintsurg-2020-SNIS.22}

Introduction Carotid artery stenting (CAS) is a safe and effective treatment for extracranial carotid artery atherosclerotic disease. While dual antiplatelet therapy (dAPT) is the standard of care following CAS, the optimal dAPT regimen and duration has not been established.

Methods We canvassed a large national healthcare claims database (IBM MarketScan) to identify patients receiving either carotid endarterectomy (CEA) or CAS for the primary indication of either ischemic stroke or carotid artery stenosis between 2007 and 2016. At least 6-months of continuous post-stent health care plan enrollment was required for inclusion. We performed univariable and multivariable regression methods to evaluate the impact of covariates on post-CAS stroke-free survival. Aggregate post-discharge antiplatelet (P2Y12 inhibitor) prescriptions were based on National Drug 